\title{
The Antecedents of Creative Performance: A Literature Review and Research Agenda
}

\author{
Zhenxing Gong \\ School of Business \\ Liaocheng University \\ Liaocheng, China \\ zxgong163.com
}

\author{
Shuo Wang \\ School of Business \\ Liaocheng University \\ Liaocheng, China \\ 37582528qq.com
}

\author{
Zanzan Zhao* \\ School of Business \\ Liaocheng University \\ Liaocheng, China \\ 1052887341qq.com \\ *Corresponding author \\ Haoyun $\mathrm{Yu}$ \\ School of Business \\ Liaocheng University \\ Liaocheng, China \\ 1686926382qq.com
}

\begin{abstract}
Creative performance is the main source of promoting innovation, enhancing competitiveness and getting competitive advantage of enterprises. Although the formation mechanism of creative performance has been researched for a long time, the antecedents that drive creative performance still remain ambiguous, and the formation mechanism is still a black box. Firstly this paper points out the problems of the antecedents' research on creative performance. Then, by reviewing of current research, this paper finds that researcher solve these problems from the concept' focus, research level, research perspective. At last, putting forward the future research direction and indicate the way for the study of the creative performance by analyzing the difficulties existing in current research.
\end{abstract}

Keywords—creative performance; creativity; antecedent

\section{INTRODUCTION}

In the rapidly changing business environment, an enterprise needs to bring forth ideas incessantly if it wants in a leading position in the competition. And creative performance is the main source of promoting innovation[1], enhancing competitiveness and getting competitive advantage of enterprise competition. And creative performance is the main source of promoting innovation[1], enhancing competitiveness and getting competitive advantage of enterprises. So creative performance becomes the outcome variable which is different from traditional performance outcome variable in the field of Organizational Behavior Science investigation, and then, it is paid close attention to by people rapidly. How to improve creative performance becomes the internal need of business administration. Component theory presented by Amabile provided the theoretical for the antecedents of creative performance investigation in organizations. Interactive theory presented by Woodman, Sawyer and Griffin pointed out creative performance is influenced by the interaction between

This research was supported by grants from the National Natural Science Foundation Youth Science Fund of China(71801120), the Ministry of Education of Humanities and Social Science Research youth fund project(18YJC630038), Shandong Social Science Planning Fund Youth Program(18DGLJ02) personal and situational factors. Then, the empirical study to check out the influence increase gradually.

Although there are a lot of theoretical and empirical studies on the causes of creative performance in the existing literature, there are still some ambiguities and shortcomings: (1) Although creative performance is mostly regarded as the first stage of innovation, it pays more attention to the generation of novel and useful ideas or viewpoints, while innovation pays more attention to ideas and viewpoints. The result of point execution is [3]. However, researchers point out that there is a continuum of multiple levels within creative performance, and previous studies have not carefully distinguished the process, confusing the factors that affect the production of innovative ideas [4].

(2) Previous studies have paid more attention to the individual level of creative performance, ignoring the study of group and organizational creative performance, resulting in limitations in the application of the results. As a form of organizational innovation at different levels, group-level creative performance can affect the level of innovation of the whole enterprise [5], and enterprise-level creative performance can affect the innovation of the whole industry, region and even the country. Although creative performance is a concept of homogeneity and isomorphism at different levels, how the factors affecting individual creative performance affect group and organizational creative performance is a problem to be solved. (3) Different scholars have different effects and internal mechanisms on the interaction of individual and situational factors on creative performance. For example, external supportive environment stimulates internal motivation, and internal motivation positively affects creative performance. This effect is stronger when employees show more initiative motivation [2]. Some studies have shown that supportive leadership feedback environment can promote the transformation of external motivation to internal motivation, and improve individual goal self-consistency. The degree of 
creativity promotes the improvement of creative performance, which is more [6] for creative personality.

In view of the above problems, different scholars from different perspectives, such as conceptual concerns, research levels, research perspectives, and so on, put forward different ideas to solve the causes of creative performance problems. This paper systematically reviews the new progress of creative performance theory and empirical research since 2000. Starting from the concept and measurement, through systematic review and analysis of the research, it excavates the dilemma and causes of the research, and puts forward solutions and future research directions. By summarizing and analyzing the antecedents of creative performance, the research results enrich the research ideas of the antecedents of creative performance, and have certain theoretical significance: first, deconstruct the formation process of creative performance from different concerns and different research perspectives; second, explore the relationship between individuals and creative performance from the perspective of cross-level research. How situational factors interact on creative performance that helps enhance organizational competitiveness. From a practical point of view, the conclusions of this study can guide enterprises to shape specific situations, cultivate relevant competencies, and screen innovative individuals, so as to improve the organizational competitiveness of creative performance.

\section{THE CONCEPT AND MEASUREMENT OF CREATIVE PERFORMANCE AT INDIVIDUAL LEVEL AND DIFFERENT CONCERNS}

The study of creative performance in organizations originates from creativity research in the field of psychology. In order to better reflect the results of creativity in the organization, many scholars use the concept of creative performance instead of creativity $[5,6]$ to highlight the value of creativity in the organization more vividly.

Although most researchers believe that creative performance involves the novelty and usefulness of products, there are great differences in the specific concepts of creative performance. Some focus on the creator, some on the way and process of creation, and some on the product and environment created. Amabile defines creative performance as innovative, practical products, processes, methods, and ideas that are produced at the individual level of employees and are of value to the organization. Although this definition has been approved by many scholars, it is often overlooked or endowed with many concepts in the study of creative performance or only focuses on some aspects in the measurement. For example, Plucker, Beghetto, and Dow's meta-analysis of 90 articles on creative performance indicated that only $38 \%$ of the articles gave the concept of creative performance, while other articles pointed out very vaguely that the concept of controversy [11]. Due to the controversy over the concept of creative performance, the corresponding measurement is also very different, [11, 12]. A meta-analysis of creative performance indicated that the most frequently used tools were the questionnaires developed by Zhou and George. Twelve percent of the studies used the questionnaires, eight percent used the questionnaires developed by Oldham and Cummings, and six percent used the questionnaires developed by Tierney, Farmer and Graen. In terms of evaluation methods, $24 \%$ of the studies used self-evaluation in measuring individual creative performance, $7 \%$ at the group level and $14 \%$ at the cross-level. In the past 10 years, there has been a tendency to change the evaluation methods to others, such as leadership evaluation [6], colleague evaluation [14].

Kaufmann pointed out that the current concept of creative performance is too broad; it should distinguish the source of innovation and innovation results, and adopt different measurement methods and develop corresponding measurement tools. Due to the different concerns of different researchers, the concept and measurement of creative performance are different, and previous studies neglected the observability and influence of creative performance. For the hard-to-observe, low-impact creative performance, there will be cognitive bias if external evaluation is adopted, and it is difficult to observe the creative performance with great influence in a short time[14]. Therefore, we need to differentiate creative performance from a more microscopic point of view, focusing not only on observable and influential creative performance, but also on difficult to observe and less influential creative performance. The study of Beghetto and Kaufman gives a more subtle way of classification. They argue that creative performance research should focus not only on "big C" (rare creativity that excels in creativity and can have a significant impact on others) and "little C" (everyday creativity, adaptability to change, and everyday problem solving), but also on "mini C" (including individuals). Creative processes of knowledge and understanding and "pro-c" (Creative skills are skills acquired through hard work that produce creativity in many fields, between small C and large C) [16].

Creative performance exists as a continuum from incremental adjustment to fundamental breakthrough. Most individuals will first perceive their own "mini-C", in the process of training knowledge and understanding of teachers, parents played a certain role in stimulating. After the encouragement and trial and error of innovation, the individual "mini C" can rise to "small C". Individuals can feel the passion of individuals in the process of realizing creativity in daily life. After receiving professional education and training, the individual can obtain the "former $\mathrm{C}$ " in one aspect, although the individual in one aspect only reaches the "small C"level, in other professional areas may have reached the "former $\mathrm{C}$ " level. After years of hardship, individuals may reach the level of 'big C" or difficult to achieve that level. The concept of creative performance in everyday work is different from that of "small $C$ ". It covers a wide range of levels of creative performance from "mini C" to "small C" to "front C". This also shows that almost everyone has creative performance in another way. Creative performance is a continuum. But this is the [18] ignored in previous studies. Compared with previous studies, the 4C model of creative performance has made a more detailed discussion on measurement, professional field, motivation mechanism, and the formation mechanism of mental illness. As can be seen from TABLE I, different creative performances differ in the above aspects, but previous studies have not distinguished, resulting in inconsistent results. 
TABLE I. THE CORRESPONDING PROBLEMS OF 4C MODEL OF CREATIVE PERFORMANCE (KAUFMAN, \& BE GHETTO, 2009)

\begin{tabular}{|c|c|c|c|c|}
\hline & Mini C & Small C & Front C & Big C \\
\hline Measurement method & $\begin{array}{l}\text { Self-evaluation, } \\
\text { analysis }\end{array}$ & $\begin{array}{l}\text { External evaluation or two-way } \\
\text { evaluation }\end{array}$ & $\begin{array}{l}\text { Two way evaluation, award and } \\
\text { honor }\end{array}$ & $\begin{array}{lll}\begin{array}{l}\text { Historic } \\
\text { honors. }\end{array} & \text { rewards and } \\
\end{array}$ \\
\hline $\begin{array}{l}\text { Professional or general } \\
\text { field }\end{array}$ & All & 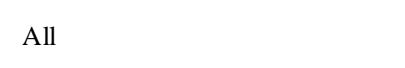 & Mostly in the professional field. & Professional field. \\
\hline Best motivation & Internal motivation & Internal motivation & Internal and external motivation & $\begin{array}{l}\text { Internal and external } \\
\text { motivation }\end{array}$ \\
\hline $\begin{array}{l}\text { Relationship with mental } \\
\text { illness }\end{array}$ & No & Yes, but very few. & In some areas there will be & In some areas there will be \\
\hline Principal investigator & Mark Runco & Ruth Richards & Greg Feist & Dean Simonton \\
\hline
\end{tabular}

From the above analysis of the concept and measurement of creative performance, we can see that the concept and measurement of creative performance vary with the changes of researchers' concerns. At present, most of the research on creative performance focuses on creative performance in daily work, which is essentially a continuum of multiple levels of creative performance from "mini C" to "small C" to "front C". At different stages of the continuum, there are different concepts, measurements and influencing factors, so it is necessary to consider more complex factors in the process of research on the precursors of creative performance at the individual level to highlight the formative differences between more specific creative performances.

\section{THE ANTECEDENTS OF CREATIVE PERFORMANCE AT DIFFERENT LEVELS}

Conceptually, creative performance can be defined and theoretically constructed at multiple levels, both as a process and as a result, and is meaningful at different levels of analysis [19]. For example, creative performance as a result is a novel and valuable product or service produced by an individual, a group or an organization, while creative performance as a process is a step or action to create creative performance, and the creative performance process is also realized at the above three levels. Creative performance at different levels shows the characteristics of isomorphism and homology. On the one hand, the structure of creative performance will not change because of cross-level changes, the emergence of new methods as a core concept throughout the individual, group and organization three levels, in order to complete the work, individuals can develop a new product or put forward new ideas, groups, organizations can also be. On the other hand, creative performance is a homologous concept, and the factors that affect individual creative performance will also have an impact on group and organizational creative performance [20]. Therefore, researchers have studied the antecedents of creative performance at different levels.

For the individual level of creative performance, researchers from the individual itself, leadership and colleagues, work situation factors in three areas were studied. One is the individual's own factors. Some studies have explored the relationship between Big Five personality factors and creative performance, and have shown that the role of personality traits on creative performance is influenced by situational factors. For example, Raja and Johns' studies show that neuroticism and extroversion are negatively correlated with creative performance, openness is positively correlated with creative performance, and conscientiousness and easiness are not correlated with creative performance when overall job characteristics are high. Some studies explored the role of individual growth demand intensity and self-efficacy [21]. Tierney and Farmer showed that the increase of creative self-efficacy positively affected the increase of creative performance, the degree of creative role identity, and perceived that Leaders' expectations for innovation increased [22]. The two is leadership and colleague factors. Leadership is the core variable affecting creative performance. Transactional leadership has a negative impact on creative performance when employees' psychological empowerment level is high [23]. Some studies also examined the effect of peer behavior on creative performance. Madjar et al. (2002) showed that peer support was conducive to employee creative performance, and when peers were seen as role models for creativity, peer care had a positive impact on creative performance. The three is work situation factors. Some studies examined the effects of time stress on creative performance, but the results were inconsistent. For example, Ohly and Fritz found that time stress had a positive effect on creative performance, while Baer and Oldham had an inverted U-shaped relationship between time stress and creative performance when they had high support for innovation and experience openness[24]. About feedback, most of the research divides the feedback into positive feedback and negative feedback, task feedback and ability feedback, information feedback and control feedback according to the valence, object and mode of the dichotomy[6]. For example, some studies believe that negative feedback can hinder employees' creative performance, but some researchers have found that negative feedback can help improve employees' creative performance. However, some studies have examined the impact of feedback environment on creative performance. The results show that feedback environment can further improve creative performance by improving employees' goal self-consistency[6].

For the group level of creative performance, researchers from the team size, team diversity, team members and other aspects of change were studied. The first is team size. Research suggests that team size is positively correlated with the diversified skills of team members to complete their work. The larger the team size, the more new ideas [25]. However, some researchers point out that too large a team will lead to social degradation and hinder the creation of creative performance. The two is team diversity. Research shows that team member diversity and creative performance are positively correlated, but there are also studies found that team member diversity and team creative performance is significantly negative correlation. Bell and colleagues argue that the contradiction arises because 
previous studies have overlooked that team diversity can be divided into shallow and deep diversity [26]. After distinguishing shallow diversity from deep diversity, HU lsheger et al. found that there was a negative correlation between shallow diversity and creativity, and a weak positive correlation between deep diversity and team creative performance. The three is the change of team members. Hirst's study found that the relationship between team members' changes and team creative performance was regulated by team members' working hours. Specifically, the change of team members had a negative impact on the team with stable members, but a weak positive impact on the team with short working hours [27].

For the creative performance at the organizational level, the researchers found that organizational innovation climate is the psychological cognition and experience of the organizational level that employees have an impact on the cultivation and development of their innovative ability. It is the consistent explanation of organizational innovation support and innovation orientation. Innovation climate plays an important intermediary role between individual and organizational innovation. Use. Organizational structure is the mode of division, cooperation and joint operation among different working departments in an organization. Research shows that centralized, rigid, bureaucratic organizational structure and lack of coordination among departments have a negative impact on organizational creative performance [28].

In terms of influencing mechanism, the researchers discussed three aspects: motivation, emotion and cognition. Most studies on creative performance are based on internal motivation. The theory of creative ingredients holds that external motivation destroys internal motivation. However, it has been revised in recent years. It holds that external incentives may not weaken internal motivation and creativity under certain circumstances. Therefore, some studies combine internal motivation with external motivation and find that feedback environment can further improve creative performance by improving employees' goal self-consistency level [6]. Regarding emotion, many studies have focused on the effect of emotion on creative performance, but failed to achieve consistent results. The Amabile et al. study suggests that positive emotions promote creative performance. George and Zhou's study suggests that negative feedback can also positively affect creative performance in situations of high reward, emphasis on creativity and clarity of feeling [29]. In terms of cognition, researchers believe that the cognitive mechanism of creative performance is a specific field, not a general intelligence factor.

The above-mentioned factors at different levels can affect creative performance at different levels, but whether the factors at one level affect creative performance at other levels have the same direction and mechanism remains to be tested. For example, HU lsheger et al. found that the positive effect of team size on creative performance was only at the group level. For individuals, the larger the team size, the more energy they spent on maintaining team interaction [3].
IV. FACTORS INFLUENCING CREATIVE PERFORMANCE FROM THE PERSPECTIVE OF NEW RESEARCH

Amabile's (1988) composition theory and Woodman's (1993) interaction theory are the two main theoretical models of creative performance research. Because these two theories regard individuals as independent individuals, they neglect that the process of individual creative performance is produced in the process of human interaction, and cultural differences will bring about different results for creative performance, which is a very risky behavior result[30]. In recent years, many scholars have carried out research from different perspectives to solve the above problems, which plays an active role in expanding the application scope of component theory and interaction theory, increasing the practicability of the theory and exploring the causes of creative performance in many aspects.

Interactive perspective. Hargadon and Bechky have shifted the research perspective of creative performance from within individuals to between individuals, shifting the focus from within individuals to interaction between individuals to explore how to solve creative problems. Through a survey of employees in professional service companies, through observation, interviews, informal conversations and file data, and from the perspective of interaction among members, four interrelated social interactions affecting creative performance are identified: seeking help, giving help, behavioral change and strengthening. High-seeking team can help each other through members to lead to changes in innovation behavior, and the establishment and help and change based on the cycle of dynamic innovation on the members will continue to influence, and then show high creative performance, team internal information exchange can better promote the promotion of creative performance. It is difficult for employees to master all the knowledge, abilities and skills produced by creative performance, and to identify the creative places in their own knowledge and experience. By sharing information and seeking information to achieve behavioral changes, they can promote the production of creative performance and improve the ability needed for creativity. The theory changes the management and research perspective of creative performance from the identification and management of individual creative performance to the understanding of social factors and the development of interaction. It shifts the focus from constant situational variables to constantly changing situational variables and from the perspective of interaction and dynamics to the rationale of creative performance. On contributing [31].

Social perspective. Perry-Smith and Shauey focus on the development of creative performance from a social perspective, emphasizing the important role of others in generating creative ideas. Based on social network theory, this paper describes the migration process of individuals from the edge of the network to the center of the network, and finds that weak relationships are more conducive to creative performance than strong ones. The marginal position with many network externalities may be related to more creative insights and groundbreaking progress, but once a high level of creative performance is achieved, marginal individuals begin to migrate gradually to the central position. The heterogeneity of knowledge and information resources attached to this centrality helps individuals to burst out new ideas and form more creative insights. This reciprocal 
and spiraling process of increasing centrality and creative performance will continue until a low return point is reached. Individuals may be too stubborn or immersed in the status quo at this point, limiting their creative performance unless they maintain relationships outside their social sphere [32].

Cultural perspective. The production mechanism of creative performance in different cultures has a very important impact on the application of management measures and the development of international business economy. At the individual level, it focuses on the moderating effect of work and social context on the impact of individual values (individualism or collectivism, distance of rights, avoidance of uncertainty) on creative performance. At the group level, the study finds that the control of team internal motivation has different effects on team creative performance in different cultures. In Eastern culture, paternalism increases team internal motivation and creative performance, while in the West, it decreases team internal motivation and consequently creative performance. The cyclic model of creative performance shows the influence of multiculturalism on organizational creative performance [1].

\section{FUTURE RESEARCH PROSPECTS}

Previous studies have explored the concept, measurement and formation mechanism of creative performance, which plays an important role in understanding and grasping creative performance. However, there are inevitably some deficiencies in the existing research, which need to be improved in the future. The specific analysis is as follows.

(1) The concept, measurement and research objects of creative performance need to be refined. Creative performance exists as a continuum from incremental adjustment to fundamental breakthroughs. Although most scholars have accepted that the concepts are highly inclusive, they ignore the hard-to-detect and/or low-impact creative performance, which the researchers define as the creative performance of small $\mathrm{C}$ and mini $C$ levels. Efficiency is the initial stage of creative performance. How to give a more precise definition and develop relative measurement tools is an urgent problem to be solved in future research. At the same time, most of the previous research objects focused on part-time college students or industrial enterprises, especially research and development personnel. In fact, the personnel engaged in innovative work in modern organizations are not limited to industrial enterprises, but also need creative performance in the fields of agricultural technology research and development, creative service industry and so on. However, previous studies on creative performance of different industries and departments lack of targeted attention, ignoring the distinction between the work content of different research objects, making the conclusions of the study lack of generalization.

(2) Trengthen cross level analysis of factors that influence creative performance. From the existing literature, we can see that the research on creative performance has paid much attention to the antecedent variables at the individual level. Because of the difficulty of collecting data by multi-level analysis and the lack of multi-level research theory in the field of creative performance research, there are two problems in the research, which will become the multi-level analysis in the future. The main objectives. On the one hand, how different levels of antecedents affect creative performance together. Among the antecedents of creative performance discussed above, individual difference is an individual level factor, and some situational variables can be considered as individual level and higher level analysis. However, in the existing literature, it is not clear how individual factors affect team and organizational creative performance, and how high-level factors affect individual creative performance. Whether this effect will change because of the stage of creative performance process. Future studies need to examine individual and higher-level context variables, and how individual differences at individual levels interact with creative performance in a common domain. On the other hand, whether the same prediction variables affect different levels of creative performance in the same way. At present, few studies provide a good example for future cross level research. Liu, Chen, and Yao examined the effects of autonomous support based on different levels of autonomy on individual creative performance with data from individual, group, and organization levels. The results showed that harmonious work passion played a fully mediating role in the influence of team autonomy support and team member autonomy orientation on creative performance of members [33]. Future research can further identify key factors at the individual, team and organizational levels, and study their impact on creative performance at three levels.

(3) Expand research on important areas that affect creative performance disputes. Firstly, there are great differences on the impact of positive or negative emotions on creative performance. The reason for this difference is that previous studies focused only on static emotion but ignored the influence of emotional change. Any factor affecting cognitive change and increasing variation may promote creative performance, and emotion is one of the sources of variation. Emotional change includes abundant information processing and individual cognition, which leads to different behaviors of individuals. Complex emotions and dynamic emotions in different contexts reflect the real state of individuals in daily life work. It is very important for organizations to carry out how to improve their creative performance in different contexts from this perspective [29]. Secondly, feedback helps employees understand the criteria of creative performance, learn the skills and methods of creativity, and stimulate the motivation of creativity. In order to reveal the relationship between feedback and creative performance accurately, future research should consider feedback as a multi-dimensional structure from an integrated perspective, and continue to explore how feedback affects the future. Creative performance and different creative performance stages give feedback [6] results. Third, whether reward is to promote or restrict creative performance is not a new problem. Most previous studies on the role of rewards have been conducted in behavioral laboratories, which are different from the workplace environment and influence the relationship between rewards and creative performance. In practice, employees work and contribute to the organization in exchange for monetary compensation, and the role of expectations and norms may have an impact on the outcome. Future research should include 
more research in the laboratory and workplace to clarify the role of incentives in employee creative performance.

(4) Pay attention to the role of cultural differences in creative performance research. At present, most of the antecedent studies on creative performance are conducted in the United States or Europe, and there are relatively few studies in eastern countries. It is necessary to strengthen the role of cultural differences in creative performance research from an international perspective. The antecedents of creative performance in different cultural and national workplaces are different. Social situational culture model points out that the influence of social situation composed of leaders, colleagues and social networks on individual, group and organizational creative performance is moderated by social culture (collectivism, consistency, conservatism). The future research on creative performance in non-Western countries and the comparative study between East and West is also an important direction [13].

(5) Explore ways to improve the creative performance that can generate positive effects. Previous research on creative performance is based on the assumption that creative performance can significantly improve performance, promote the effectiveness of team work, and have a positive impact on organizational change and success. But some scholars are concerned that creative performance is not always positive. Creative performance implies the risk of conflict. When creative individuals face obstacles from their superiors and colleagues who are satisfied with the status quo, they will suffer emotional frustration, conflict aggravation and deterioration of working relationships. Gino and Ariely argue that highly creative individuals may resort to immoral means when seeking solutions to tasks[1]. Research on the dark side of creative performance shakes the assumption that there is no discrimination between the positive effects of creative performance and helps us understand the "multifaceted" creative performance phenomenon in the workplace. Future research should construct positive and negative models of creative performance to show that an integrated model outlines these interrelationships, including the sources, processes, and outcomes of creative performance. Are there different antecedents for the creative performance process and the creative performance result of negative results, and are there differences between the process and the result itself, thus affecting the effectiveness of the results? Under what circumstances, creative performance is beneficial or harmful to individuals, groups and organizations? Should creative performance be performed or abandoned if it is beneficial to the organization and harmful to the individual or other individuals in the organization? How can managers maximize their creative performance incentives by avoiding the "dark side" of the dilemma? These are questions worth discussing.

\section{REFERENCES}

[1] Zhou J, Shalley C E. Expanding the scope and impact of organizational creativity research[J]. Handbook of organizational creativity. 2008, 28: 125-147.

[2] Grant A M, Berry J W. The necessity of others is the mother of invention: Intrinsic and prosocial motivations, perspective taking, and creativity[J]. Academy of Management Journal. 2011, 54(1): 73-96.
[3] Hülsheger U R, Anderson N, Salgado J F. Team-level predictors of innovation at work: a comprehensive meta-analysis spanning three decades of research.[J]. Journal of Applied psychology. 2009, 94(5): 1128.

[4] Lingo E L, O'Mahony S. Nexus Work: Brokerage on Creative Projects[J]. Administrative Science Quarterly. 2009, 55(1): 47-81.

[5] Sue-Chan C, Hempel P S. The Creativity-Performance Relationship: How Rewarding Creativity Moderates the Expression of Creativity[J]. Human Resource Management. 2016, 55(4): 637-653.

[6] Zhang J, Gong Z, Zhang S, et al. Impact of the Supervisor Feedback Environment on Creative Performance: A Moderated Mediation Model[J]. Frontiers in Psychology.2017, 8(1): 256.

[7] Harrison S H, Rouse E D. An Inductive Study of Feedback Interactions over the Course of Creative Projects[J]. Academy of Management Journal. 2015, 58(2): 375-404.

[8] Liu L, Wang L, Ren J, et al. Promotion/prevention focus and creative performance: Is it moderated by evaluative stress? [J]. Personality \& Individual Differences. 2017, 105: 185-193.

[9] Jonathan A P, Ronald A B, Gayle T D. Why Isn't Creativity More Important to Educational Psychologists? Potentials, Pitfalls, and Future Directions in Creativity Research[J]. Educational Psychologist. 2004, 39(2): 83-96.

[10] Liu Xinmei, Bai Yang, Zhang Ruili. Connotation and measurement of organizational creativity [J]. soft science 2011, 25(4): 60-62.(In Chinese)

[11] Alge B J, Ballinger G A, Tangirala S, et al. Information privacy in organizations: empowering creative and extrarole performance.[J]. Journal of Applied Psychology. 2006, 91(1): 221.

[12] Beghetto Ronald A.,Kaufman James C. The genesis of creative greatness: mini-c and the expert performance approach[J]. High Ability Studies. 2007, 18(1): 59-61.

[13] Beghetto R A, Kaufman J C. Do we all have multicreative potential?[J]. ZDM. 2009, 41(1): 39-44.

[14] Mar B, Inés H. Team Creative Environment as a Mediator Between CWX and R\&D Team Performance and Moderating Boundary Conditions[J]. 2017.

[15] Chen G, Mathieu J E, Bliese P D. A framework for conducting multilevel construct validation[J]. Research in multilevel issues: Multilevel issues in organizational behavior and processes. 2004, 3: 273-303.

[16] Raja U, Johns G. The jo int effects of personality and job scope on in-role performance, citizenship behaviors, and creativity[J]. human relations. 2010.

[17] Tierney P, Farmer S M. Creative self-efficacy develop ment and creative performance over time.[J]. Journal of Applied Psychology. 2011, 96(2): 277.

[18] Pieterse A N, Van Knippenberg D, Schippers M, et al. Transformational and transactional leadership and innovative behavior: The moderating role of psychological empowerment[J]. Journal of Organizational Behavior. 2010, 31(4): 609-623.

[19] Baer M, Oldham G R. The curvilinear relation between experienced creative time pressure and creativity: moderating effects of openness to experience and support for creativity.[J]. Journal of Applied Psychology. 2006, 91(4): 963.

[20] Derosa D M, Smith C L, Hantula D A. The medium matters: Mining the long-promised merit of group interaction in creative idea generation tasks in a meta-analysis of the electronic group brainstorming literature[J]. Computers in Human Behavior. 2007, 23(3): 1549-1581.

[21] Bell S T, Villado A J, Lukasik M, et al. Getting specific: A meta-analysis of the team demographic diversity and performance relationships[J]. Journal of Management. 2011, 37: 709-743.

[22] Hirst G, Van Knippenberg D, Zhou J. A cross-level perspective on employee creativity: Goal orientation, team learning behavior, and individual creativity[J]. Academy of Management Journal. 2009, 52(2): 280-293.

[23] Hunter S T, Bedell K E, Mumford M D. Dimensions of Creative Climate[J]. The International Journal of Creativity \& Problem Solving. 2005, 15(2): 97-116. 
[24] Bledow R, Rosing K, Frese M. A dynamic perspective on affect and creativity[J]. Academy of Management Journal. 2013, 56(2): 432-450.

[25] Bai Yang, Liu Xinmei. Formation mechanism of organizational creativity [J]. soft science. 2013, 27(6): 25-28.(In Chinese)

[26] Hargadon A B, Bechky B A. When collections of creative become creative collectives: A field study of problem solving at work[J]. Organization Science. 2006, 17(4): 484-500.

[27] Perry-Smith J E, Coff R W. In the mood for entrepreneurial creativity? How optimal group affect differs for generating and selecting ideas for new ventures[J]. Strategic Entrepreneurship Journal. 2011, 5(3): 247-268.

[28] Liu D, Chen X P, Yao X. From autonomy to creativity: a multilevel investigation of the mediat ing role of harmonious passion.[J]. Journal of Applied Psychology.2011, 96(2): 294-309.

[29] Anderson N, Potočnik K, Zhou J. Innovation and creativity in organizations a state-of-the-science review, prospective commentary, and guiding framework [J]. Journal of Management. 2014, 40(5): 1297-1333. 